\title{
Online Monitoring and Control of Upstream Cell Culture Process Using 1D \& 2D-LC with SegFlow Interface
}

\author{
Letha Chemmalil $^{1}$, Dhanuka Wasalthanthri ${ }^{1}$, Xin Zhang ${ }^{1}$, June Kuang ${ }^{1}$, Chun Shao ${ }^{1}$, \\ Robin Barbour ${ }^{2}$, Sohil Bhavsar ${ }^{1}$, Tanushree Prabhakar ${ }^{1}$, Ryan Knihtila ${ }^{3}$, Jay West $^{1}$, Neha \\ Puri $^{1}$, Kyle McHugh ${ }^{1}$, Matthew Rehmann ${ }^{4}$, Qin $\mathrm{He}^{4}$, Jianlin Xu ${ }^{5}$, Michael Borys ${ }^{5}$, Julia \\ Ding ${ }^{1}$, and Zheng Jian $\mathrm{Li}^{5}$ \\ ${ }^{1}$ Bristol-Myers Squibb Co \\ ${ }^{2}$ University of Rochester \\ ${ }^{3}$ Dragonfly Therapeutics \\ ${ }^{4}$ Bristol-Myers Squibb \\ ${ }^{5}$ Bristol-Myers Squibb Company
}

January 4, 2021

\begin{abstract}
The biopharmaceutical industry is transitioning from currently deployed batch-mode bioprocessing to a highly efficient and agile next generation bioprocessing with the adaptation of continuous bioprocessing, which reduces the capital investment and operational costs. Continuous bioprocessing, aligned with FDA's quality-by-design (QbD) platform, is designed to develop robust processes to deliver safe and effective drugs. With the deployment of knowledge based operations, product quality can be built into the process to achieve desired critical quality attributes (CQAs) with reduced variability. To facilitate next generation continuous bio-processing, it is essential to embrace a fundamental shift-in-paradigm from "quality-by-testing" to "quality-by-design", which requires the deployment of process analytical technologies (PAT). With the adaptation of PAT, a systematic approach of process and product understanding and timely process control are feasible. Deployment of PAT tools for real-time monitoring of CQAs and feedback control is critical for continuous bioprocessing. Given the current deficiency in PAT tools to support continuous bioprocessing, we have integrated Agilent 2D-LC with a post-flow-splitter in conjunction with the SegFlow automated sampler to the bioreactors. With this integrated system, we have established a platform for online measurements of titer and CQAs of monoclonal antibodies (mAbs) as well as amino acid concentrations of bioreactor cell culture.
\end{abstract}

\section{Hosted file}

Manuscript._Dec._2020.pdf available at https://authorea.com/users/297575/articles/502399online-monitoring-and-control-of-upstream-cell-culture-process-using-1d-2d-lc-withsegflow-interface 